\title{
Plasticité cérébrale et neuropathologies Nouvelles voies pour le médicament
}

$>$ Il est désormais acquis que la plasticité neuronale est importante, même chez l'adulte, pour la Michael Spedding, Pierre Lestage formation de nouvelles connexions comme pour celle de nouveaux neurones (neurogenèse) ou de cellules gliales. Cependant, le vieillissement et les facteurs de stress peuvent altérer cette plasticité et favoriser le développement d'une atrophie cérébrale. Des anomalies de plasticité neuronale pourraient être l'un des facteurs contribuant au développement des maladies neuropsychiatriques, et ce aux différents stades de la vie. La compréhension de ces processus, l'étude de leur implication dans la pathologie neuropsychiatrique et la recherche d'agents pharmacologiques à visée thérapeutique susceptibles de moduler cette plasticité neuronale constitue un des thèmes centraux de la recherche en neurosciences au sein de l'Institut de Recherches Servier.<

La plasticité cérébrale, au cours du développement mais aussi chez l'adulte, est bien plus importante qu'il n'était admis il y a quelques années. En stimulant une vibrisse de souris éveillée pendant 24 heures, G.W. Knott et al. [1] ont mis en évidence une augmentation de $36 \%$ de la plasticité synaptique dans l'unité corticale correspondante, et une augmentation parallèle des synapses excitatrices et inhibitrices s'accompagnant d'un accroissement important de la connectivité en réponse à un stimulus persistant. Cette modification dépend de la production d'une neurotrophine, le brainderived neurotrophic factor (BDNF). C'est le changement le plus important observé dans le cerveau adulte mais, dans le cerveau en développement, le nombre de connexions double quotidiennement dans l'hippocampe, par exemple chez le rat. Inversement, une stimulation à basse fréquence peut, dans certains cas, entraîner une atrophie.

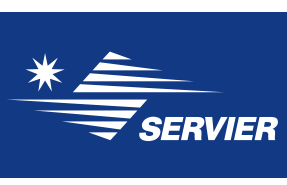

Institut de Recherches

Servier,

11 , rue des Moulineaux,

Plasticité moléculaire 92150 Suresnes, France. et cellulaire

La modification à long terme de l'efficacité de la transmission synaptique est l'un des mécanismes de base de la mémoire et de l'apprentissage. L'activation synchrone des éléments pré- et post-synaptiques provoque une augmentation de l'efficacité de la transmission synaptique, ou «potentialisation à long terme » (PLT). D'un point de vue cellulaire ou moléculaire, la plasticité neuronale est rapide et étendue, avec l'induction d'une potentialisation à long terme entraînant des modifications morphologiques des épines dendritiques (Figure 1). Cette plasticité chez l'adulte est observée dans les structures cérébrales impliquées dans la mémoire: systèmes hippocampique (mémoire déclarative, gestion de contexte), amygdalien (gestion de la peur et des émotions) et thalamocortical (mémoire procédurale) (Figure 2). La potentialisation à long terme dépend fondamentalement des concentrations en calcium dans les zones présynaptiques et les épines dendritiques. Ainsi, de fortes concentrations calciques entraînent l'activation de la protéine kinase II dépendante du complexe calcium/calmoduline (CaMKII) et la phosphorylation de nombreuses protéines cibles impliquées dans les bases moléculaires de cette plasticité (Figure I et Encadré).

Les récentes évolutions conceptuelles sur la neurogenèse adulte (production de nouveaux neurones fonctionnellement actifs) ont de vastes implications pour les maladies neuropsychiatriques. La neurogenèse diminue progressivement avec l'âge, et les facteurs de stress accentuent ce déclin. G. Kempermann et al. [2] ont montré qu'un enrichissement environnemental tout au long de la vie peut entraîner une multiplication par 5 de la neurogenèse hippocampique chez la souris âgée. 


\section{Modification des systèmes cérébraux}

Le nombre de synapses est particulièrement dynamique pendant le développement du cerveau puis, chez l'adulte, dans les aires associées à l'apprentissage et à la mémoire, y compris émotionelle (Figure 2). La plasticité cérébrale est donc très importante, et sensible au stress et au vieillissement. Une potentialisation à long terme dans l'hippocampe induit une potentialisation à long terme dans le cortex préfrontal, d'une manière entièrement dépendante de la libération de dopamine [3]. Cependant, un stress aigu bloque la potentialisation à long terme dans l'hippocampe chez le rat [3], ainsi que la mémoire hippocampique [4], indiquant un lien potentiel entre l'inhibition de la fonction hippocampique liée au stress et une réduction de l'activité du cortex préfrontal. Ces effets sont exacerbés par le vieillissement, qui est associé à une sensibilité accrue à une atrophie induite par le stress [4]. Ainsi, la potentialisation à long terme survenant dans l'hippocampe, qui induit celle du cortex préfrontal, est un index de la plasticité synaptique dans ces deux aires du cerveau susceptibles de présenter une atrophie en cas de dépression ou de schizophrénie (voir plus loin). Cette potentialisation à long terme est extrêmement sensible au stress (Figure 3). Un stress chronique (d'une durée de trois semaines) reconfigure l'arborisation dendritique des neurones dans l'hippocampe et dans ses zones de projection dans le cortex préfrontal [5]. En parallèle, l'enrichissement environnemental augmente rapidement la densité des épines dendritiques, favorisant la potentialisation à long terme et élevant les concentrations des neurotrophines telles que le BDNF.

\section{Plasticité neuronale et désordres psychiatriques}

L'impact de périodes prolongées d'activation ou de dépression neuronale au niveau des différentes voies neuronales entraînera finalement des effets trophiques ou une atrophie dans les régions cérébrales impliquées. Ces phénomènes ont été étudiés à l'aide de la mesure du débit sanguin cérébral, de l'imagerie par résonance magnétique (IRM), de la visualisation de variations du volume cérébral et de l'anatomohistopathologie.

La dépression peut survenir à tout âge, et l'on observe une comorbidité fréquente avec l'anxiété et les troubles liés au stress. Les troubles psychiatriques sont associés, dans au moins un tiers des cas, à des problèmes survenus au cours du développement et de l'enfance $[6,7]$. Sur la base de nombreuses études sur le rôle des facteurs de stress dans le développement, M.J. Meaney [7] donne à la synaptogenèse hippocampique un rôle central pour apprendre à gérer le stress. Les environnements très stressants produisent des systèmes hippocampiques moins développés, mais une augmentation de la plasticité dans l'amygdale.

On observe fréquemment une réduction du débit sanguin cérébral dans les zones frontales lors des états dépressifs [8]. Une dépression prolongée et récurrente peut être associée à une atrophie marquée du cortex et de l'hippocampe. Des études post-mortem ont mis en évidence une atrophie corticale, avec une réduction de la taille des neurones et de la densité neuronale et gliale, dans les cortex orbitofrontal et frontal dorsolatéral [9]. Les études d'imagerie ont révélé que l'atrophie de l'hippocampe chez les patients dépressifs pouvait être associée au stress et à une dépression récurrente $[10,11]$. La dépression est également associée à une altération significative de la mémoire de travail, avec un impact sur les fonctions cognitives. H.S. Mayberg [8] a apporté des arguments convaincants en faveur d'une réduction du débit sanguin cérébral dans le cortex cingulaire antérieur pendant un épisode dépressif, et de la restauration du débit sanguin dans cette aire après un traitement par la paroxetine mené avec succès. Cette aire est directement innervée par l'hippocampe, justifiant l'hypothèse d'un 
dysfonctionnement du cortex hippocampofrontal après un stress aigu, qui peut se tranformer en une atrophie en cas de trouble chronique. J.D. Bremner et al. [12] ont mis en évidence une réduction de $32 \%$ du volume de la zone orbitofrontale médiale chez les patients déprimés. La plasticité neuronale pourrait donc jouer un rôle clé dans le développement des troubles neuropsychiatriques, d'autant que les zones à risque sont les zones où la plasticité neuronale est altérée par les facteurs de stress (Figure 3 ).

\section{Médicaments entravant l'impact du stress sur la plasticité neuronale ou modulant l'activité synaptique et la production de neurotrophines}

\section{Inhibition par la tianeptine (Stablon ${ }^{\circledR}$ ) de l'atrophie neuronale induite par le stress Une abondante littérature étaye l'hypothèse d'une activité thérapeutique des antidépresseurs liée à leur inhibition de la recapture des monoamines. Cependant, la dépression n'est pas toujours très bien traitée: un tiers des patients ne réagit pas aux médicaments, et un tiers réagit au placebo. \\ La tianeptine (Stablon ${ }^{\circledR}$ ), un antidépresseur efficace} chez l'homme, possède un mécanisme d'action différent de celui des antidépresseurs classiques. En l'absence d'hypothèse a priori sur son mode d'action, ses effets sur l'expression génique ont été explorés: ils indiquent une incidence sur la plasticité synaptique, la concentration en protéine présynaptique Mss4 (mammalian supressor of sec4), par exemple, étant augmentée par les traitements antidépresseurs, notamment la tianeptine, et réduite par le stress [13]. Nous avons focalisé ces recherches, chez l'animal, sur les zones à risque chez l'homme pendant le stress et la dépression (hippocampe/cortex médio-préfrontal).

En administration aiguë, la tianeptine a une action importante sur la potentialisation à long terme dans l'hippocampe et le cortex préfrontal (Figure 3). Chez le rat, le stress aigu provoqué par une exposition sur une plate-forme élevée entraîne une réduction directe de la potentialisation à long terme dans la région CAl de l'hippocampe et dans les zones de projection vers le cortex préfrontal, régions cérébrales essentielles pour la gestion du contexte. L'administration de faibles doses de tianeptine, après le stress, restaure totalement la potentialisation à long terme, dans l'hippocampe et le cortex préfrontal, alors que la fluoxétine est peu efficace [14]. Ces résultats indiquent que la tianeptine restaure la plasticité neuronale dans les zones cérébrales à risque. Le groupe de D.M. Diamond [15] a récemment montré que la potentialisation à long terme est augmentée par le stress dans l'amygdale, mais que la tianeptine ne module pas cette augmentation, indiquant que la gestion de contexte pourrait être améliorée et les réponses émotives laissées intactes.

Les conséquences structurelles d'un stress chronique ont été évaluées au cours d'une collaboration avec le groupe de McEwen (New York, ÉtatsUnis) [4]. Un tel stress induit une atrophie de l'arborisation dendritique de la région hippocampique $\mathrm{CA} 3$ et dans les zones de projection préfron-

tales chez le rat. Ces effets sont contrecarrés par la tianeptine [16], mais pas par la fluoxétine; un excès de glucocorticoïdes entraîne également des changements histomorphologiques au niveau de l'hippocampe, effets également contrecarrés par la tianeptine. Chez ces animaux, la tianeptine inverse les déficits cognitifs induits par le stress [4], de même que ceux causés par une dénervation septohippocampique partielle [17]. La tianeptine entraîne une inversion de l'atrophie hippocampique évaluée par IRM, ainsi que de l'inhibition de la neurogenèse causée par un stress chro- 
nique chez le tupaïa (tree shrew) [18]. La tianeptine exerce donc un effet bénéfique aigu sur la potentialisation à long terme, un effet chronique sur l'arborisation dendritique et un effet sur la mémoire, en un mot sur la plasticité neuronale altérée par le stress.

La tianeptine agit directement, car des effets ont été observés sur des tranches hippocampiques examinées in vitro et ex vivo [19], notamment une augmentation des courants glutamatergiques AMPA/NMDA ( $\alpha$-amino-3-hydroxy-5-méthyl-isoxazole/N-méthyl-D-aspartate) par phosphorylation. Chez le rat, un stress de contention augmente l'expression des transporteurs de glutamate (GLT-1) spécifiquement dans la zone où la reconfiguration dendritique est la plus intense (la partie CA3 de l'hippocampe), et cette augmentation est contrecarrée par la tianeptine [5]. Des antidépresseurs tels que la tianeptine pourraient donc agir directement sur la plasticité neuronale via les systèmes glutamatergiques, ce qui ouvre une nouvelle voie de recherche.

\section{Modulateurs allostériques des récepteurs AMPA}

Les modulateurs allostériques positifs des récepteurs AMPA modifient la plasticité neuronale et favorisent la potentialisation à long terme et la mémoire, en ralentissant la désensibilisation des récepteurs AMPA activés par un neurotransmetteur endogène, le glutamate. De plus, ces molécules augmentent le taux de neurotrophines, particulièrement du BDNF. Des essais cliniques sont actuellement en cours sur plusieurs composés, dont le S 18986, issu de la Recherche Servier.

Les premiers résultats publiés par I. Ito et al. [20] indiquaient que l'aniracetam, à très hautes concentrations, ralentissait la désensibilisation des récepteurs AMPA, expliquant ainsi les effets promnésiants du composé. En s'appuyant sur ces résultats, Servier a mis au point le S 18986, un modulateur allostérique puissant qui facilite la potentialisation à long terme hippocampique et améliore les processus mnésiques hippocampiques (test de conditionnement passif bloqué par la scopolamine, test de reconnaissance d'objet) [21, 22]. En parallèle, les chercheurs de Cortex Pharmaceuticals ont développé le CX516 [23], précédant d'autres sociétés qui se dirigent elles aussi vers des essais cliniques.
Des études ont également montré que les modulateurs allostériques positifs des récepteurs AMPA augmentaient la synthèse et la libération du BDNF [24], qui a des effets trophiques et neuroprotecteurs. Les modulateurs allostériques positifs AMPA montrent des effets neuroprotecteurs et neurotrophiques qui pourraient être sous-tendus par ces propriétés [25]. Des substances présentant des effets trophiques chez I'homme devraient s'avérer bénéfiques à long terme. Ce domaine est d'un intérêt potentiel considérable pour les pharmacologues, car une pharmacologie différente (désensibilisation contre désactivation) peut être escomptée de composés présentant une sélectivité pour les différents sous-types de récepteurs et une efficacité différente au niveau des récepteurs AMPA. Les cibles thérapeutiques actuelles sont la schizophrénie, la dépression et les troubles cognitifs dans la maladie d'Alzheimer. En facilitant la transmission glutamatergique et en favorisant la plasticité cérébrale, ces substances pourraient représenter un nouveau principe thérapeutique, si les essais cliniques s'avèrent positifs.

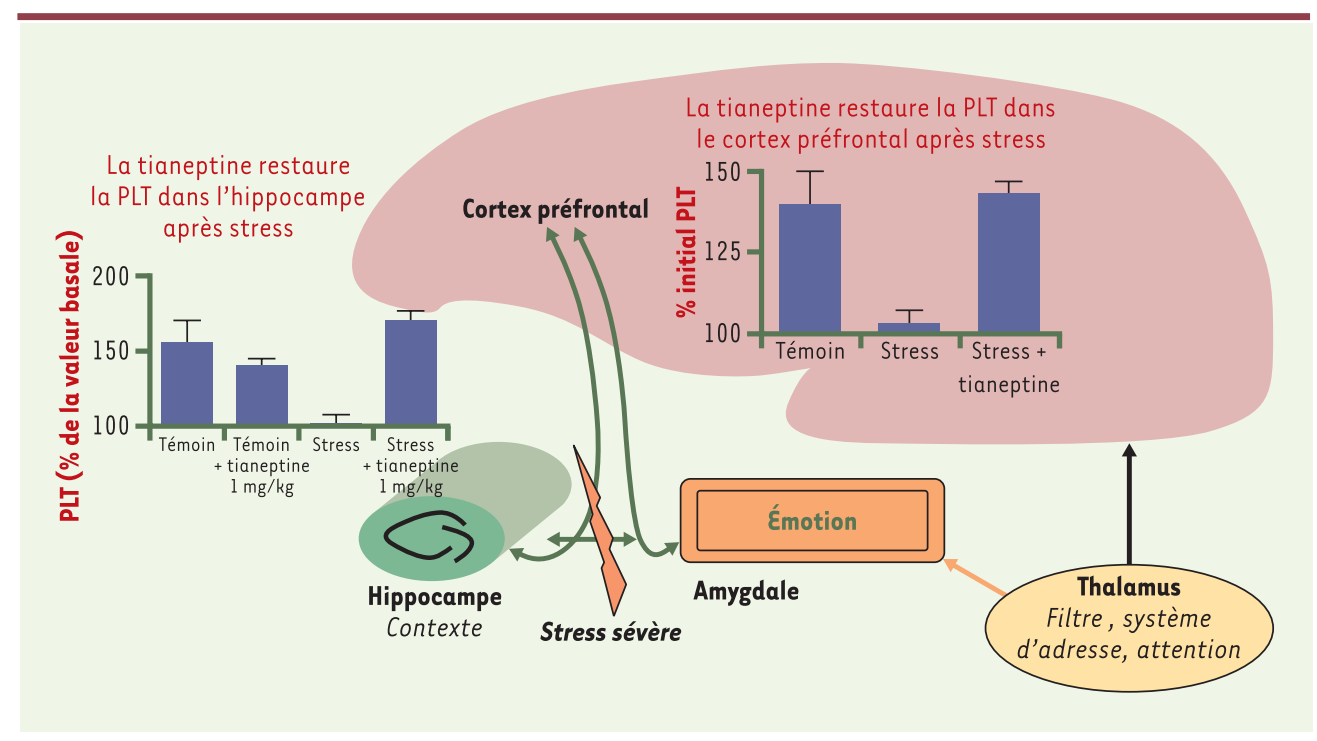

Figure 3. Interaction du cortex préfrontal avec l'amygdale et l'hippocampe, et impact du stress. De manière simpliste, l'hippocampe est essentiel pour évaluer le contexte (spatial et temporel) et fournit la «couleur » émotionnelle (même si le cortex joue également un rôle majeur dans ce cadre). Chez le rat, un stress aigu (exposition sur une plate-forme élevée pendant 30 minutes) bloque la potentialisation à long terme (PLT) pendant plusieurs heures dans l'hippocampe et le cortex préfrontal, zones impliquées dans la genèse de la dépression. L'administration de tianeptine ( $1 \mathrm{mg} / \mathrm{kg}$ par voie intrapéritonéale) après le stress restaure complètement la PLT; ce produit rétablit la plasticité synaptique précisément dans les zones du cerveau à risque; en revanche, la fluoxétine est peu active dans ce test $[3,14]$. 


\section{Conclusions}

Certains dogmes insistaient sur le fait que l'activité des antidépresseurs était due à l'inhibition de la recapture des neurotransmetteurs monoaminergiques (sérotonine, noradrénaline). La Recherche Servier a cependant montré qu'un antidépresseur, la tianeptine, agit chez l'homme par l'intermédiaire d'autres mécanismes, ciblés sur les zones du cerveau à risque dans la dépression, mais dépourvus des effets secondaires classiquement associés aux inhibiteurs de recapture de la sérotonine. De plus, de nouveaux produits tels que les modulateurs allostériques positifs des récepteurs AMPA agissent sur la mémoire et la plasticité neuronale en partie via des effets neurotrophiques. De nouvelles générations de produits sont en évaluation préclinique et clinique, avec l'espoir d'agir directement sur les causes des maladies psychiatriques et neurologiques. $\diamond$

\section{SUMMARY}

\section{Synaptic plasticity}

and neuropathology:

new approaches in drug discovery

Neuronal plasticity is now known to be very important in the adult, both in the formation of new synaptic connections and of new neurones (neurogenesis) and of glial cells. However, old age and stress can inhibit this plasticity and lead to cerebral atrophy. The time course of changes in neuronal plasticity involves, in the first milliseconds to seconds, changes in synaptic strength (long term potentialisation, LTP, or long term depression, LTD), then, over minutes to hours, changes in the number of synaptic connections (linked to changes in neurotrophic factors), and over weeks to months, to changes in neuronal reconfiguration. These changes in brain systems are particularly targeted in psychiatric disorders to the areas which are sensitive to stress and play roles in memory and emotion (hippocampus, amygdala and prefrontal cortex). The discovery and

\section{MÉCANISMES DE MODULATION DE LA PLASTICITÉ NEURONALE}

\author{
(voir aussi Figure 3)
}

Transport des récepteurs AMPA ( $\alpha$-amino-3-hydroxy-5-méthyl-isoxazole) et NMDA (Nméthyl-D-aspartate) vers et hors de la membrane cellulaire synaptique : ce phénomène est crucial pour le passage du courant, qui est augmenté en cas d'incorporation dans la synapse (potentialisation à long terme), et diminué (dépression à long terme) en cas de diffusion des récepteurs vers la membrane extrasynaptique [26] ou d'internalisation [27].

Augmentation du calcium dans les compartiments pré- et post-synaptiques: I'augmentation postsynaptique a été particulièrement bien modélisée et est, en outre, responsable des changements de forme des épines dendritiques.

Fréquence: en dehors des trains d'impulsions à haute fréquence qui l'induisent, la potentialisation à long terme est favorisée par le rythme $\theta(4,5-8 \mathrm{~Hz})$, qui résulte en une phosphorylation du résidu y 876 de la sous-unité GluR2 des récepteurs AMPA. II s'agit d'un mécanisme capital, car le rythme $\theta$ coordonne les cellules de lieu dans l'hippocampe et constitue l'un des principaux rythmes દદG impliqués dans la cognition. Le rythme $\theta$ est attribué à l'hippocampe mais, comme l'a souligné G. Buszaki [28], il s'agit d'un rythme qui unifie tout le système limbique. Une stimulation à basse fréquence $(<2 \mathrm{~Hz})$ induit une DLT et entraîne une internalisation des récepteurs AMPA. II est évident que la précision de l'arrivée des potentiels d'action dans un réseau neuronal est capital, changeant la plasticité du système et les calculs du réseau.

Stress aigu: ce phénomène bloque la potentialisation à long terme dans l'hippocampe et le cortex préfrontal (Figure 2) [3, 14]), mais facilite la potentialisation à long terme dans l'amygdale (partie du cerveau importante pour l'apprentissage de la peur [29]). Cela se traduit, lors de stress chroniques, par des changements morphologiques et des atrophies hippocampiques [4] et préfrontales.

Phosphorylation des récepteurs AMPA et NMDA: ce mécanisme est important en terme d'internalisation/externalisation des récepteurs et de passage du courant. Des sites de phosphorylation multiples sont responsables d'effets trophiques, avec des mécanismes reposant sur I'AMP cyclique, le GMP cyclique, la calcium/calmoduline kinase II ou la protéine kinase $C$. Les systèmes de neurotransmission peuvent donc affecter directement la plasticité, et c'est l'un des principaux mécanismes permettant à la dopamine, à la noradrénaline, à la 5-hydroxytryptamine et à l'acétylcholine d'exercer leurs effets trophiques. Neurotrophines, cytokines et potentialisation à long terme: il a récemment été suggéré que des augmentations de facteurs neurotrophiques, notamment du BDNF (brain-derived neurotrophic factor), jouaient un rôle critique pour le mode d'action des antidépresseurs [11]. De nombreux travaux ont fait état de l'effet trophique et neuroprotecteur du BDNF, et de son rôle dans la plasticité synaptique [24, 25]. Ce facteur est essentiel à la potentialisation à long terme, car celle-ci est entravée chez des souris invalidées pour son gène. Le BDNF régit donc à la fois la survie sélective des neurones pendant le développement cérébral et la régulation, fondée sur l'expérience, de l'activation synaptique tout au long de la vie. Le BDNF module l'arborisation axonale pour favoriser la croissance, tandis que l'activité neuronale participe à la stabilisation des branches d'axones, les deux signaux convergeant pour modeler la forme des dendrites axonales. Cependant, il est important de souligner que les neurotrophines pourraient agir de concert ou de manière antagoniste et que, souvent, les études ne mesurent qu'une seule neurotrophine.

La potentialisation à long terme augmente-t-elle le nombre de connexions neuronales? La potentialisation à long terme peut être suivie par une division des épines dendritiques menant à un doublement du nombre de connexions synaptiques [30]. La littérature est partagée à ce sujet, mais il est possible qu'une disparition de synapses puisse compenser toute augmentation de ce nombre, et que les modifications puissent être très rapides [30]. La rétraction des épines est un processus rapide entraînant une réduction des épines dendritiques liée au phénomène de dépression à long terme. Ainsi, il pourrait exister un contrôle homéostatique du nombre de synapses s'opposant à un mécanisme hebbien d'amplification provoqué par l'activité. 
development of drugs modifying neuronal plasticity and neurotrophins production has been a priority for Servier research for the last ten years; Servier has a clinically effective antidepressant, tianeptine $\left(\right.$ Stablon $\left.^{\circledR}\right)$, with a favourable side effect profile, but which does not inhibit the uptake of serotonin, or other monoamines. However, this drug can reverse the deleterious effects of stress on neuronal plasticity, thereby acting on the causes of psychiatric disorders. Furthermore, a new research area is being investigated - facilitation of AMPA receptors, favouring the production of neurotrophic factors. $\diamond$

\section{RéFÉRENCES}

1. Knott GW, Quairiaux C, Genoud C, Welker $\varepsilon$. Formation of dendritic spines with GABAergic synapses induced by whisker stimulation in adult mice. Neuron 2002; 34: 265-73.

2. Kempermann G, Gast D, Gage FH. Neuroplasticity in old age: Sustained fivefold induction of hippocampal neurogenesis by long-term environmental enrichment. Ann Neurol 2002; 52 : 135-43.

3. Rocher C, Spedding M, Munoz C, Jay TM. Acute stress-induced changes in hippocampal/prefrontal circuits in rats: Effects of antidepressants. Cereb Cortex 2004; $14: 224-9$.

4. McEwen BS. Stress and hippocampal plasticity. Annu Rev Neurosci 1999 22: 105-22.

5. Reagan LP, Rosell DR, Wood GE, et al. Chronic restraint stress upregulates GLT-1 mRNA and protein expression in the rat hippocampus: Reversal by tianeptine. Proc Natl Acad Sci USA 2004; 101: 2179-84.

6. Rutter ML. Psychosocial adversity and child psychopathology. $\mathrm{Br}$ J Psychiatry 1999; 174: 480-93.

7. Meaney MJ. Maternal care, gene expression, and the transmission of individual differences in stress reactivity across generations. Annu Rev Neurosci 2001; 24: 1161-92.

8. Mayberg HS. Limbic-cortical dysregulation: A proposed model of depression. J Neuropsychiatry Clin Neurosci 1997; 9: 471-81.

9. Rajkowska G, Miguel-Hidalgo JJ, Wei J, et al. Morphometric evidence for neuronal and glial prefrontal cell pathology in major depression. Biol Psychiatry 1999; 45: 1085-98.

10. Sheline YI, Sanghavi M, Mintun MA, Gado MH. Depression duration but not age predicts hippocampal volume loss in medically healthy women with recurrent major depression. J Neurosci 1999; 19: 5034-43.

11. Duman RS, Charney DS. Cell atrophy and loss in major depression. Biol Psychiatry 1999; 45: 1083-4.

12. Bremner JD, Vythilingam $M$, Vermetten $\varepsilon$, et al. Reduced volume of orbitofrontal cortex in major depression. Biol Psychiatry 2002; 51 : 273-9.

13. Andriamampandry C, Muller C, Schmidt-Mutter C, et al. Mss4 gene is upregulated in rat brain after chronic treatment with antidepressant and down-regulated when rats are anhedonic. Mol Pharmacol 2002; 62: 1332-8.
14. Shakesby AC, Anwyl R, Rowan MJ. Overcoming the effects of stress on synaptic plasticity in the intact hippocampus: Rapid actions of serotonergic and antidepressant agents. J Neurosci 2002; 22: 3638-44.

15. Diamond DM, Campbell A, Park CR, Vouimba RM. Preclinical research on stress, memory and the brain in the development of pharmacotherapy for depression. EurJ Neuropsychopharmacol 2004; 14: S491-5.

16. Cassano GB, Heinze G, Lôo H, et al. A double-blind comparison of tianeptine, imipramine and placebo in the treatment of major depressive episodes. Eur Psychiatry 1996; 11: 254-9.

17. Morris RG, Kelly S, Burney D, et al. Tianeptine and its enantiomers: Effects on spatial memory in rats with medial septum lesions. Neuropharmacology 2001; 41: 272-81.

18. Czeh B, Michaelis T, Watanabe T, et al. Stress-induced changes in cerebral metabolites, hippocampal volume, and cell proliferation are prevented by antidepressant treatment with tianeptine. Proc Natl Acad Sci USA 2001; 98: 12796-801.

19. Kole MH, Swan L, Fuchs $\varepsilon$. The antidepressant tianeptine persistently modulates glutamate receptor currents of the hippocampal CA3 commissural associational synapse in chronically stressed rats. Eur J Neurosci 2002; 16: 807-16.

20. Ito I, Tanabe S, Kohda A, Sugiyama H. Allosteric potentiation of quisqualate receptors by a nootropic drug aniracetam. J Physiol (Lond) $1990 ; 424: 533-43$

21. Lebrun C, Pilliere $\varepsilon$, Lestage P. Effects of S 18986-1, a novel cognitive enhancer, on memory performances in an object recognition task in rats. EurJ Pharmacol 2000; 401: 205-12.

22. Lockhart B, lop F, Closier M, Lestage P. (S)-2,3-dihydro[3,4]cyclopentano-1,2,4-benzothiadiazine-1,1-dioxide: (S18986-1) a positive modulator of AMPA receptors enhances (S)-AMPA-mediated ${ }^{3} \mathrm{H}$ noradrenaline release from rat hippocampal and frontal cortex slices. EurJ Pharmacol 2000; 401: 145-53.

23. Arai AC, Xia YF, Rogers G, et al. Benzamide-type AMPA receptor modulators form two subfamilies with distinct modes of action. $J$ Pharmacol Exp Ther 2002; 303: 1075-85.

24. Lauterborn JC, Lynch G, Vanderklish P, et al. Positive modulation of AMPA receptors increases neurotrophin expression by hippocampal and cortical neurons. J Neurosci $2000 ; 20: 8-21$.

25. Dicou $E$, Rangon CM, Guimiot F, et al. Positive allosteric modulators of AMPA receptors are neuroprotective against lesions induced by an NMDA agonist in neonatal mouse brain. Brain Res 2003; 970 : 221-5.

26. Tardin C, Cognet L, Bats C, et al. Direct imaging of lateral movements of AMPA receptors inside synapses. EMBOJ 2003; 22: 4656-65.

27. Barry MF, Ziff $\varepsilon B$. Receptor trafficking and the plasticity of excitatory synapses. Curr Opin Neurobiol 2002; 12: 279-86.

28. Buzsaki G. Theta oscillations in the hippocampus. Neuron $2002 ; 33$ 325-40.

29. Rogan MT, Staubli UV, LeDoux JE. Fear conditioning induces associative long-term potentiation in the amygdala. Nature 1997; 390: 604-7.

30. Marrone DF, Petit TL. The role of synaptic morphology in neural plasticity : structural interactions underlying synaptic power. Brain Res Brain Res Rev 2002; 38: 291-308.
TIRÉS À PART

M. Spedding 